Check for updates

Cite this: RSC Adv., 2018, 8, 761

Received 12th October 2017 Accepted 11th December 2017

DOI: 10.1039/c7ra11269f

rsc.li/rsc-advances

\title{
Large-size and high performance visible-light photodetectors based on two-dimensional hybrid materials SnS/RGO
}

\author{
Renfu Zhuo, (D)* Shiyong Zuo, Weiwei Quan, De Yan, Baisong Geng, Jun Wang \\ and Xuehu Men
}

\begin{abstract}
We report a facile solvothermal method to synthesize two-dimensional hybrid materials consisting of layered SnS nanosheets and reduced graphene oxide (SnS/RGO). Large-size photodetectors with a channel length/width $=2 \mathrm{~mm} / 7 \mathrm{~mm}$ are fabricated on $\mathrm{Si} / \mathrm{SiO}_{2}$ substrates, showing an excellent photoresponsivity of $0.18 \mathrm{~A} \mathrm{~W}^{-1}$ under visible-light illumination with a detectivity of $4.18 \times 10^{10}$ Jones, as well as fast rise and decay times $\left(\tau_{\text {rise }}=\tau_{\text {decay }}=0.4 \mathrm{~s}\right)$. SnS/RGO hybrids are therefore promising candidates for potential applications in optoelectronics and low cost, high performance, and reliable photodetectors.
\end{abstract}

\section{Introduction}

The development of 2-dimensional (2D) graphene and layered metal chalcogenide nanosheets has created extensive interest in photodetection in the past few years because of their high carrier mobility, convenient material fabrication and compatibility with semiconductor electronics., ${ }^{\mathbf{1 2}}$ For photon energy utilization purposes, the optically active materials should not only have high absorption coefficient through the whole solar spectrum, but also have the ability to suppress the fast electronhole recombinations. ${ }^{3-5}$ Therefore numerous attempts have been made to combine these $2 \mathrm{D}$ nanosheets with semiconductor nanostructures, this kind of hybrid photodetectors achieves ultrahigh responsivity due to the synergism between the strong absorption of semiconducting nanostructures and the extremely fast conduction of carriers within the $2 \mathrm{D}$ nanosheets. ${ }^{6-9}$ These hybrid photodetectors, such as graphene/PbS, ${ }^{6}$ graphene/ZnO, ${ }^{7} \mathrm{MoS}_{2} / \mathrm{PbS}^{8} \mathrm{SnS}_{2} / \mathrm{PbS}^{9}$ et al. have already demonstrated outstanding performance. Unfortunately, all of them are on the micron scales and the fabrication must undergo extremely complex and precise processes for example, 2D nanosheets synthesized by chemical vapour deposition must transfer onto substrates, 2D nanosheets prepared by exfoliation are all on the micron scales, and photodetectors with micro-size channel length which assembled on substrates must use photolithography, all of which are unfit for large-size device fabrication.

$\mathrm{SnS}$ is considered to be an excellent material for its layered structural-related semiconducting and optical properties. It has an absorption coefficient near the fundamental absorption edge

School of Physical Science and Technology, Lanzhou University, Lanzhou, Gansu, 730000, China. E-mail: zhuorf@lzu.edu.cn; Tel: +86-931-8912719 in the order of $10^{4} \mathrm{~cm}^{-1}$, and it can absorb almost the entire visible and the near-infrared range of the solar spectrum. ${ }^{\mathbf{1 0 - 1 3}}$ In addition, $\mathrm{SnS}$ is a low-cost, nontoxic, earth-abundant, and heavy-metal free material for optoelectronic devices, and has shown promising applications in lithium-ion batteries, ${ }^{\mathbf{1 0}}$ photodetectors ${ }^{\mathbf{1 1}}$ and photocatalysts. ${ }^{13}$ Different morphologies of SnS, such as nanoflowers, nanoribbons, nanoparticles and nanoflakes, have been synthesized on the several substrates as large-size SnS films. ${ }^{\mathbf{1 2 - 1 7}}$ The large-size SnS films based photodetectors have been established with high $I_{\text {light }} / I_{\text {dark }}$ ratio, excellent stability and fast rise and decay times. However, their photoresponsivity is still relatively low, almost at the level of $\mathrm{mA} \mathrm{W}^{-1}$. Another kind of micro-size single SnS nanoribbon/ nanosheet based photodetectors, having also been reported by several groups exhibit relatively high photoresponsivity above the level of $\mathrm{A} \mathrm{W}^{-1} \cdot{ }^{17-22}$ But such single $\mathrm{SnS}$ nanoribbon/ nanosheet based photodetectors are on the micron scales and their fabrication must undergo extremely complex and precise processes, which is not suitable for large-scale applications. Nevertheless, all of these reports show that SnS nanostructures are good candidates for high performance photodetectors.

To the best of our knowledge, SnS/RGO hybrids have rarely been reported as photodetector. Herein, we report a facile solvothermal method to synthesize the SnS/RGO hybrid nanosheets. By drop-casting $\mathrm{SnS} / \mathrm{RGO}$ hybrid nanosheets on $\mathrm{Si} / \mathrm{SiO}_{2}$ substrates as large-size photodetectors, enhanced visible-light photosensitivity is obtained.

\section{Experimental}

\section{Preparation of SnS/RGO hybrid nanosheets}

Natural graphite powder was oxidized to graphite oxide (GO) by the modified Hummers' method. The SnS/RGO hybrid 
nanosheets were prepared by a solvothermal route according to previous reports. ${ }^{10}$ In a typical synthesis of the SnS/RGO hybrid, $20 \mathrm{mg}$ of graphite oxide was dispersed into $30 \mathrm{ml}$ ethylene glycol solution by ultra-sonication for $60 \mathrm{~min}$. Next, $226 \mathrm{mg} \mathrm{SnCl}_{2}$ $\cdot 2 \mathrm{H}_{2} \mathrm{O}(1 \mathrm{mmol})$ and $210 \mathrm{mg}$ citric acid were added to the solution and then stirred for $60 \mathrm{~min}$. Finally, $2 \mathrm{ml}\left(\mathrm{NH}_{3}\right)_{2} \mathrm{~S}$ was added to the solution, which was further stirred for $5 \mathrm{~min}$, and then transferred to a $40 \mathrm{ml}$ Teflon-lined stainless steel autoclave. After heating at $463 \mathrm{~K}$ for $18 \mathrm{~h}$ and naturally cooled to the room temperature, the black product was filtered and washed several times with deionized water and absolute ethanol, and then dried at $333 \mathrm{~K}$ for $24 \mathrm{~h}$ to obtain the two dimensional (2D) hybrid SnS/RGO.

\section{Characterization}

The morphology and structure were observed by transmission electron microscopy (TEM: JEM-2100) operated at $200 \mathrm{kV}$. The crystal structures of the as-synthesized products were characterized by X-ray diffraction (XRD) on a Philips X'Pert Pro Diffractometer. The Raman spectra were recorded with a Jobin YVON HR800UV Laser Raman spectrometer. The X-ray photoelectron spectroscopy (XPS) data were determined on Thermo Scientific XPS spectrometers.

\section{The fabrication of photodetectors}

The as-synthesized SnS/RGO hybrid nanosheets were dispersed into toluene and dropped onto Si substrates with $300 \mathrm{~nm}$ thick $\mathrm{SiO}_{2}$ layer $(1 \mathrm{~cm} \times 1 \mathrm{~cm})$, electrodes of $\mathrm{Ag}(100 \mathrm{~nm})$ using a metal mask were deposited by DC sputtering to form a channel of $L / W=2 \mathrm{~mm} / 7 \mathrm{~mm}$. The final devices were annealed at $400 \mathrm{~K}$ for $2 \mathrm{~h}$ with the protection of argon gas in order to remove residues and enhance the metallic contacts. The current-voltage $(I-V)$ characteristics were studied by using a Keithley 4200SCS-CVU semiconductor parameter analyzer. For photodetection, a broadband laser-driven light source (EQ-99, Energetiq) calibrated by an UV-enhanced silicon photodiode provided an incident light, while the current were recorded by a current meter Keithley (2410), an attenuator and a power meter to get different intensities of the incident beam, and the spot effective area was determined to be $0.01 \mathrm{~cm}^{2}$.

\section{Results and discussion}

The XRD pattern of the SnS/RGO hybrid nanosheets is shown in Fig. 1(a). Clearly, all reflection peaks can be indexed to pure SnS with orthorhombic phase (JCPDS 39-0354). No other peaks were observed in the XRD pattern, indicating that the as-synthesized products were in the pure phase. The (001) peak of GO at around $2 \theta=10.2^{\circ}$ is absent, which indicates that the present solvothermal reaction not only produces the SnS nanosheets but also simultaneously reduces the GO. Fig. 1(b) shows the Raman spectra of SnS/RGO, with the peak at $213.8 \mathrm{~cm}^{-1}$ which is related to the $\mathrm{Ag}$ vibration mode of $\mathrm{SnS}$. Also, two typical carbon peaks are observed: the $\mathrm{G}$ band $\left(1592.3 \mathrm{~cm}^{-1}\right)$ and the $\mathrm{D}$ band $\left(1349.1 \mathrm{~cm}^{-1}\right)$. As we know, the $\mathrm{G}$ band is the response of the in-plane stretching motion of symmetric $\mathrm{sp}^{2} \mathrm{C}-\mathrm{C}$ bonds, whereas the $\mathrm{D}$ band results from the disruption of the symmetrical hexagonal graphitic lattice. ${ }^{10}$

The morphology and microstructure of the as-prepared SnS/ RGO hybrids were studied by field emission scanning electron microscopy (SEM) and transmission electron microscopy (TEM). The SEM image in Fig. 2(a) reveals the 2D morphology of the SnS/RGO hybrids. Fig. 2(b) reveals the TEM image of SnS/ RGO hybrid nanosheets, it clearly shows curved SnS nanosheets dispersed on large RGO substrates. Fig. 2(c) and (d) display the high-magnification TEM image selected from Fig. 2(b). The SnS shows a well-layered structure, supported on the RGO surface. There are lattice fringes with lattice spacings of $0.28 \mathrm{~nm}$ (inset of Fig. 2(d)), corresponding to the (040) plane of SnS. ${ }^{18}$ Because of the weak van der Waals interaction, SnS shows a well-layered structure with the thickness of 2-10 nm, which indicates that each sheet is composed of 8-40 layers of SnS along the $b$-axis. This weakly bound interlayer characteristic, the nucleation and the subsequent growth of $\mathrm{SnS}$ atoms on the lattice along the $b$-axis, lead to the formation of the $\mathrm{SnS} /$ RGO hybrid nanosheets. ${ }^{18,23}$

To further evaluate the SnS/RGO hybrid nanosheets, X-ray photoelectron spectroscopy (XPS) was performed, as shown in Fig. 3(a). The peaks of Sn are shown in Fig. 3(b). It can be seen that the two strong peaks at around 486.6 and $495.0 \mathrm{eV}$ can be attributed to $\mathrm{Sn} 3 \mathrm{~d}_{5 / 2}$ and $3 \mathrm{~d}_{3 / 2}$ respectively, which agrees well with the reference data of $\mathrm{Sn}^{2+}$ in $\mathrm{SnS}$. No evidence of $\mathrm{Sn}^{4+}$ (binding energy at $485.9 \mathrm{eV}$ ) was detected in the spectra. ${ }^{\mathbf{1 1}}$ The $\mathrm{S}$ $2 \mathrm{p}_{3 / 2}$ at $161.8 \mathrm{eV}$ (Fig. 3(c)) and $\mathrm{S} 2 \mathrm{~s}_{1 / 2}$ at $223.5 \mathrm{eV}$ are attributed to the binding energies of SnS. In Fig. 3(d), the spectrum $\mathrm{C} 1 \mathrm{~s}$ can be deconvoluted into four peaks, which are ascribed to the $\mathrm{sp}^{2}$ bonded carbon $(\mathrm{C}-\mathrm{C}, 284.6 \mathrm{eV}$ and $\mathrm{C}=\mathrm{C}, 285.1$ ), the epoxy/ hydroxyl groups $(\mathrm{C}-\mathrm{O}, 286.9 \mathrm{eV})$ and the carboxyl groups $(\mathrm{C}=\mathrm{O}$, $289 \mathrm{eV})$. Under the solvothermal condition, the increased
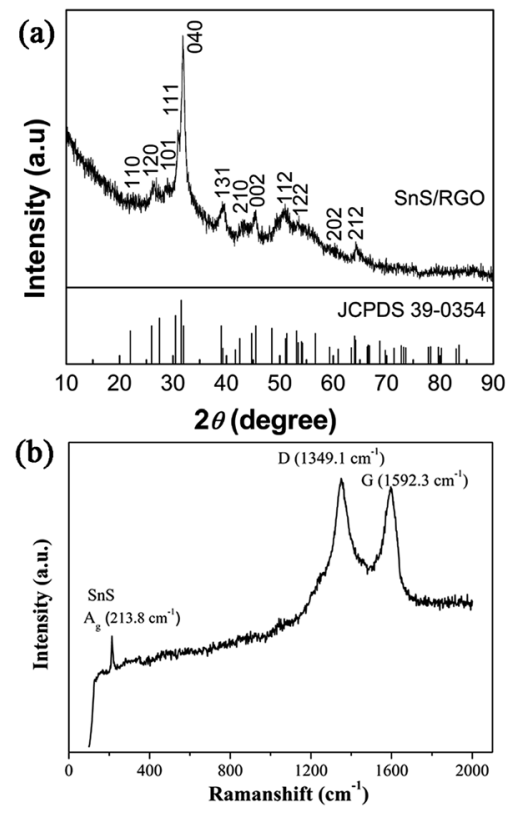

Fig. 1 (a) The XRD patterns; (b) the Raman spectra of the SnS/RGO hybrid nanosheets. 


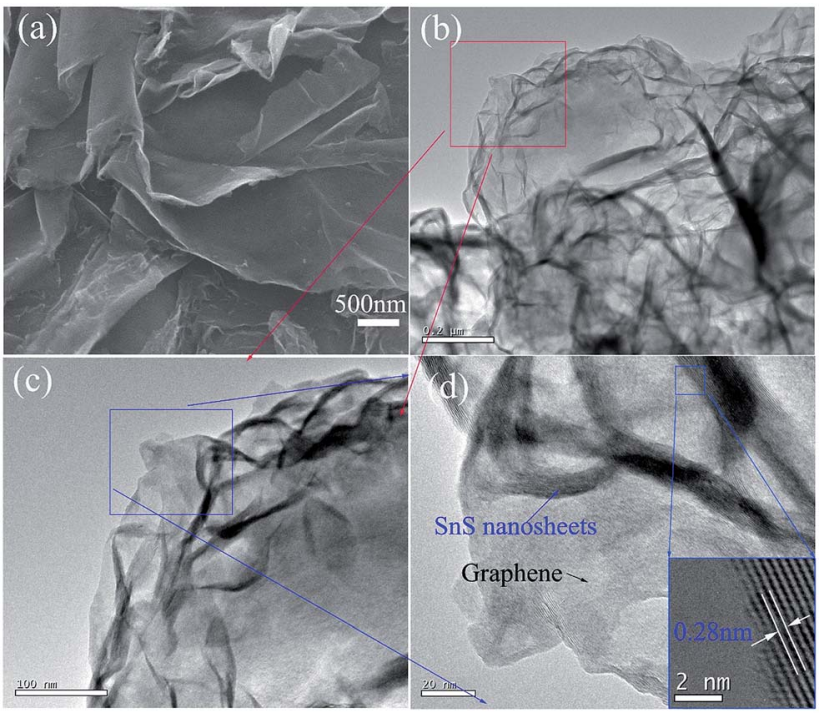

Fig. 2 (a) The SEM image; (b-d) the TEM images of the SnS/RGO hybrid nanosheets.

temperature and autogenous pressure led to the reduction of graphene oxide into $\mathrm{RGO}$, but the $\mathrm{C}-\mathrm{O}$ or $\mathrm{C}=\mathrm{O}$ of the interfacial bonding was still left after the reduction. ${ }^{10,24}$

The optical image and the schematic of the device of SnS/ RGO hybrid nanosheets based photodetector is shown in Fig. 4(a). Fig. 4(b) presents the transfer characteristics $\left(I_{\mathrm{ds}}-V_{\mathrm{g}}\right)$ of the device with SnS/RGO in the dark and under visible-light illumination $\left(P=10 \mathrm{~mW} \mathrm{~cm}{ }^{-2}, V_{\mathrm{ds}}=5 \mathrm{~V}\right)$, respectively. In comparison, the corresponding transfer curves of the (a)
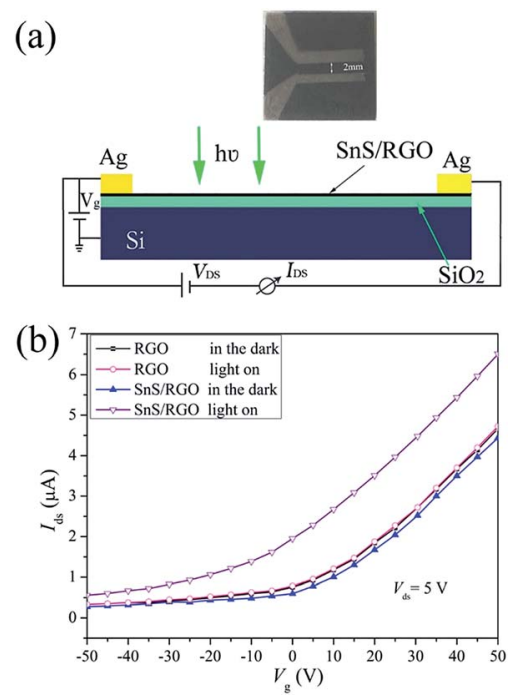

Fig. 4 (a) The optical image and schematic of a representative device. (b) The transfer characteristics $\left(I_{\mathrm{ds}}-V_{\mathrm{g}}\right)$ of the photodetectors based on $\mathrm{RGO}$ and $\mathrm{SnS} / \mathrm{RGO}$ in the dark and under visible-light illumination, $P=$ $10 \mathrm{~mW} \mathrm{~cm}^{-2}$

undecorated device with pure RGO are also plotted in Fig. 4(b). All devices exhibit typical n-type semiconducting behaviour. We find that the electrical properties of RGO after SnS decoration become very sensitive to light. To preclude the possibility of intrinsic photoresponses of RGO, we first detect the electrical properties of RGO before SnS decoration when visible light is switched on and off. We don't observe the obvious current changes under light irradiation, which set the foundation for
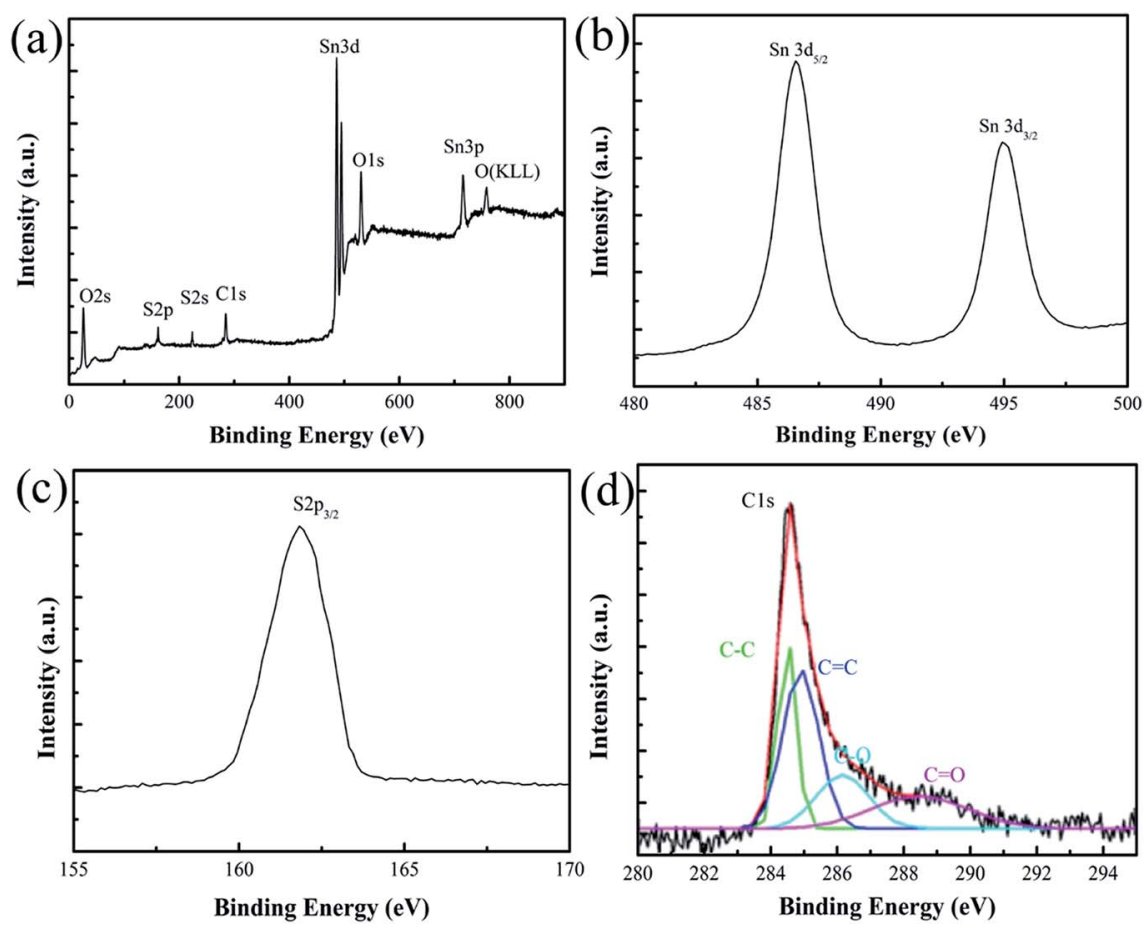

Fig. 3 XPS results of SnS/RGO hybrid nanosheets. 

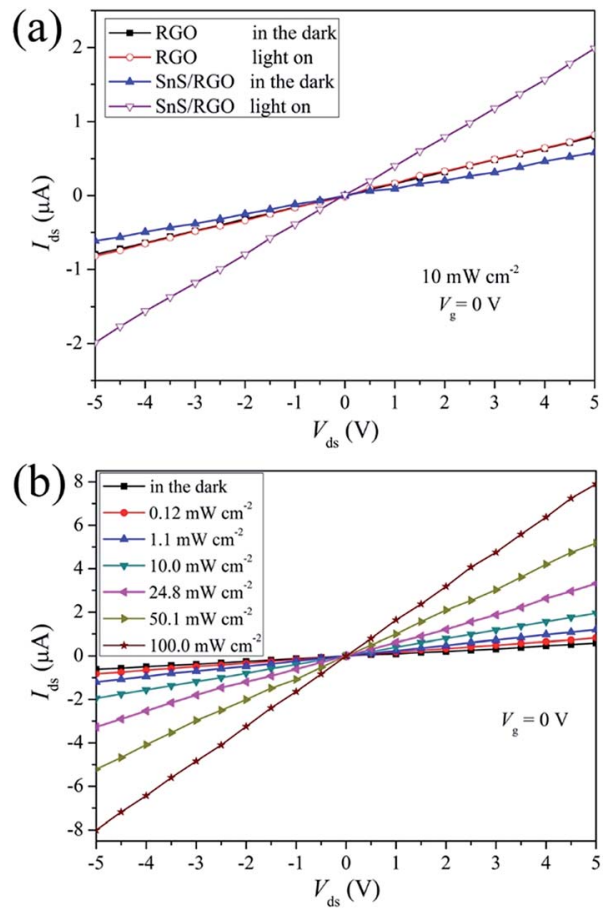

Fig. 5 (a) Output characteristics $I_{\mathrm{ds}}-V_{\mathrm{ds}}$ of the photodetectors based on RGO and SnS/RGO in the dark and under visible-light illumination. (b) $I_{\mathrm{ds}}-V_{\mathrm{ds}}$ curves of SnS/RGO with different light intensities.

the following studies of the charge transfer at the SnS/RGO interfaces. After SnS decoration, the devices show a slight conductance decrease, most likely due to the scattering effect for carriers created by SnS which are intimately covered on RGO surfaces. ${ }^{25}$ Noticeably, upon light irradiation, obvious increase
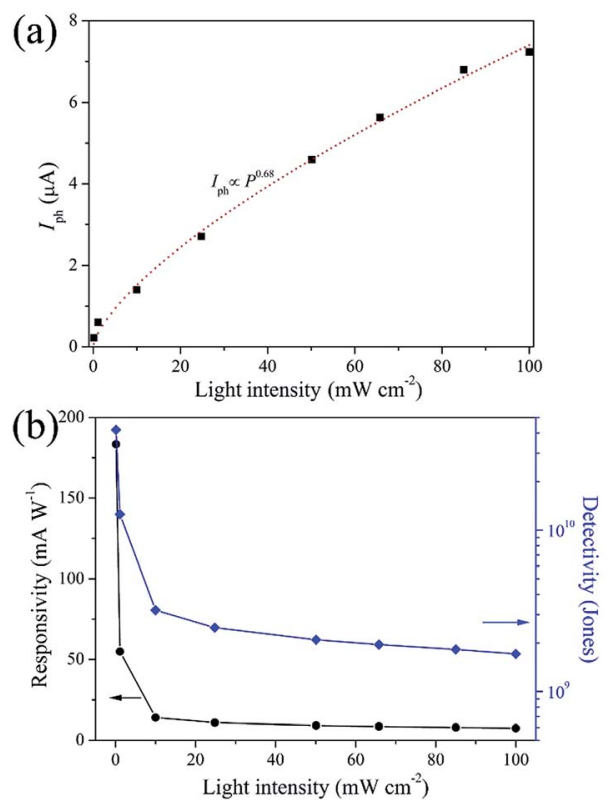

Fig. 6 (a) Plot of photocurrent, (b) corresponding responsivity and detectivity of the device versus light intensities, measured at $V_{\mathrm{ds}}=$ $5.0 \mathrm{~V}, \mathrm{~V}_{\mathrm{g}}=0 \mathrm{~V}$.
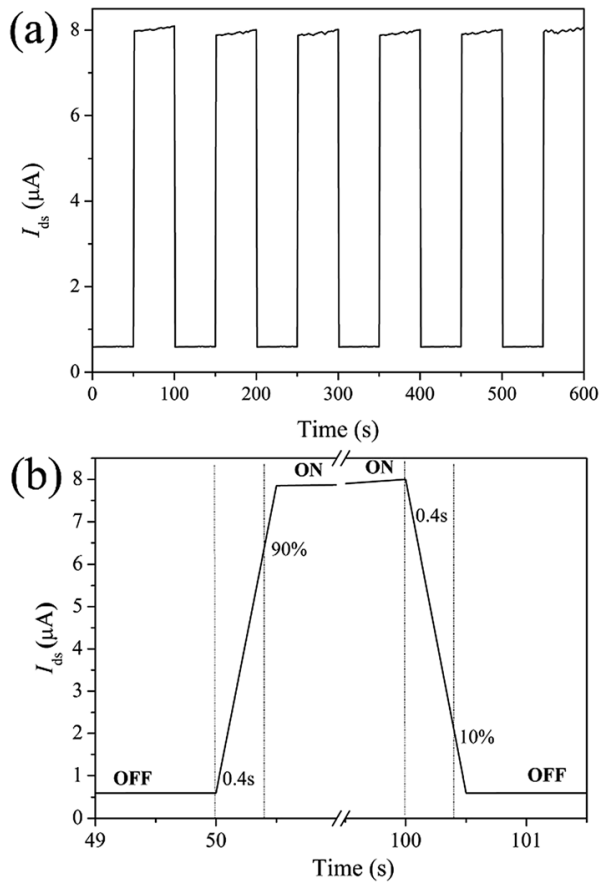

Fig. 7 (a) Time response of the SnS/RGO device illuminated with and without light $\left(100 \mathrm{~mW} \mathrm{~cm}^{-2}\right)$. (b) Rise and decay time. All measured at $V_{\mathrm{ds}}=5.0 \mathrm{~V}, V_{\mathrm{g}}=0 \mathrm{~V}$

in source-drain current $\left(I_{\mathrm{ds}}\right)$ occurred regardless of the gate bias in the device based on SnS/RGO. Previous studies have demonstrated that the incident visible light generated delocalized electron-hole pairs over the SnS nanosheets. ${ }^{11}$ The photogenerated holes behave as coulomb traps, the electrons in SnS could penetrate through the interface to the RGO conductive channel, which resulted in the n-type carrier concentration increase in the SnS/RGO hybrid devices. The mobility of the carriers can be calculated by:

$$
\mu=\frac{L}{W \times V_{\mathrm{ds}} \times C_{\mathrm{i}}} \times \frac{\mathrm{d} I_{\mathrm{ds}}}{\mathrm{d} V_{\mathrm{g}}} .
$$

where $L$ and $W$ are the length and width of the channel, $C_{\mathrm{i}}$ is the capacitance per unit area (11.4 $\mathrm{nF} \mathrm{cm}{ }^{-2}$ for $300 \mathrm{~nm} \mathrm{SiO}_{2}$ layer), $\mathrm{d} I_{\mathrm{ds}} / \mathrm{d} V_{\mathrm{g}}$ is the transconductance obtained from the slope of the plot in the linear region of $I_{\mathrm{ds}}$ versus $V_{\mathrm{g}}$ curves. ${ }^{11,26}$ The calculated $\mu$ is $\approx 5.0 \mathrm{~cm}^{2} \mathrm{~V}^{-1} \mathrm{~s}^{-1}$, which is at least one order higher than that of single SnS nanoribbon. ${ }^{11}$

Fig. 5(a) shows the output characteristics $\left(I_{\mathrm{ds}}-V_{\mathrm{ds}}\right)$ of the photodetector based on RGO and SnS/RGO in dark and under illumination $\left(P=10 \mathrm{~mW} \mathrm{~cm}^{-2}, V_{\mathrm{g}}=0 \mathrm{~V}\right)$, respectively. The plots show a linear increase of the current with the applied voltage, indicating an ohmic contact between the SnS/RGO and Ag electrodes. In addition, a pronounced increase of the photocurrent under illumination is observed after SnS decoration, suggesting a strong absorption of SnS and effective charge transfer between the SnS decoration and the RGO channel. Fig. 5(b) shows the $I_{\mathrm{ds}}-V_{\mathrm{ds}}$ curves of the SnS/RGO under illumination with different light intensities. It is found that the conductance and slopes of $I-V$ curves presented monotonic 
Table 1 Comparison of the parameters for the performance of the large-size SnS film based photodetectors

\begin{tabular}{|c|c|c|c|c|c|c|}
\hline Large-size SnS film based photodetector & Responsivity $\left[\mathrm{A} \mathrm{W}^{-1}\right]$ & Detectivity [Jones] & $\begin{array}{l}\text { Power density } \\
{\left[\mathrm{mW} \mathrm{cm}^{-2}\right]}\end{array}$ & $I_{\text {light }} / I_{\text {dark }}$ & $\begin{array}{l}\text { Rise/decay } \\
\text { time }[\mathrm{s}]\end{array}$ & References \\
\hline SnS nanoflowers on FTO & $7.05 \times 10^{-4}$ & - & 100 & - & $0.5 / 0.5$ & 12 \\
\hline SnS films on glass & $4.0 \times 10^{-4}$ & $7.1 \times 10^{7}$ & 60 & 1.32 & $1.2 / 3.0$ & 14 \\
\hline SnS nanoflakes on PET & $4.0 \times 10^{-6}$ & - & 25 & 14 & $0.53 / 0.5$ & 15 \\
\hline SnS nanoparticles on $\mathrm{Si} / \mathrm{SiO}_{2}$ & $1.7 \times 10^{-3}$ & - & 50 & 1.2 & $0.45 / 0.56$ & 16 \\
\hline
\end{tabular}

increase according to the light power density, which meant higher sensitivity of the device illuminated by higher power intensity at a higher bias voltage. A higher power intensity and higher voltage can drive photon-generated charge carriers to reach the electrodes faster, suppressing recombination of holeelectron pairs. ${ }^{21,27}$ This phenomenon suggests that the photocurrent is mainly determined by the amount of photongenerated carriers under illumination.

In Fig. 6(a), the photocurrent $\left(I_{\mathrm{ph}}=I_{\text {light }}-I_{\text {dark }}\right)$ of SnS/RGO as a function of the light intensity is plotted, measured at $V_{\mathrm{ds}}=$ $5.0 \mathrm{~V}, V_{\mathrm{g}}=0 \mathrm{~V}$. It can be seen that the device is very sensitive to the illumination power. By fitting the experimental data, it can be expressed by a power-law equation $I_{\mathrm{ph}} \propto P^{0.68}$, demonstrating the efficiency of the photogenerated charge carriers. ${ }^{26,28}$ The performance of the photodetector is generally judged by several parameters, such as the responsivity $(R)$, detectivity $\left(D^{*}\right)$, response rate, and stability. Among the above parameters $R$, defined as the amount of photocurrent generated per unit area per unit illumination intensity, can be given by $R=I_{\mathrm{ph}} / P S,{ }^{\mathbf{2 0}}$ where $S$ is the effective illumination area. According to this formula, a remarkable responsivity about $0.18 \mathrm{~A} \mathrm{~W}^{-1}(P=0.12$ $\left.\mathrm{mW} \mathrm{cm}^{-2}, V_{\mathrm{ds}}=5.0 \mathrm{~V}, V_{\mathrm{g}}=0 \mathrm{~V}\right)$ is obtained. This high photoresponsivity may result from the efficient absorption and optimized device configuration. In addition, the dependence of the photoresponsivity on the light power intensity irradiated on the device is depicted in Fig. 6(b). The phenomenon that the responsivity decreases as the light intensity increases is frequently observed in photoconductivity photodetectors. Under high-power illumination, the numbers of photogenerated carriers available for extraction will be reduced, which is caused by the Auger process or the saturation of recombination/trap states that influence the lifetime of the generated carriers. ${ }^{26}$ Moreover, considering the fact that it is impractical to compare and evaluate directly the responsivity of photodetectors fabricated from other 2D materials, unless the measurements are done under similar environmental conditions. Thus, another important parameter, detectivity, was also investigated. The specific detectivity $\left(D^{*}\right)$ defined as a measure of the least detectable radiant power, can be expressed as: ${ }^{28}$

$$
D^{*}=R \sqrt{S / 2 e I_{\text {dark }}}
$$

where $e$ is the electronic charge. The detectivity $\left(D^{*}\right)$ was estimated to be $\sim 4.18 \times 10^{10}$ Jones $\left(P=0.12 \mathrm{~mW} \mathrm{~cm}{ }^{-2}\right)$, as is depicted in Fig. 6(b).
The dependence of the current on time under alternating dark and illumination conditions $\left(100 \mathrm{~mW} \mathrm{~cm}^{-2}\right)$ with ON/OFF switch cycle of $100 \mathrm{~s}$ is shown in Fig. 7(a). The photocurrent increases to a stable value under illumination, and then dramatically decreases to its initial value as the illumination is turned off, thereby showing excellent stability and reproducible characteristics. $I_{\text {light }} / I_{\text {dark }}$ ratio of about 14 , a rise time and a decay time both of $0.4 \mathrm{~s}$ are obtained, indicating the rapid photoresponse characteristics (Fig. 7(b)).

The performance of the SnS/RGO hybrid nanosheets photodetector in comparison with previously reported large-size SnS film based devices is summarized in Table 1. It is clear that the key parameters of our device are much higher than that of SnS film based large-size photodetectors, ${ }^{12-17}$ and are also close to that of single SnS nanoribbon/nanosheet based complex micron-size photodetectors. ${ }^{\mathbf{1 1 1 8 - 2 2}}$ The enhanced photoresponse may be attributed to several reasons. The good ohmic contacts should contribute to a rapid diffusion of the charge carriers, and therefore, accelerate the transportation of carriers and increase the response speed. Furthermore, a large specific surface area of the SnS/RGO nanosheets can promote a fast, effective separation of photocarriers and defer the electron-hole recombinations simultaneously to prolong the photoexcited carrier lifetime, resulting in the high responsivity and detectivity. ${ }^{22}$ More significantly, the synergism between the strong absorption of SnS and the fast conduction of electrons within the RGO would be beneficial to the separation of electron-hole pairs and thus to the higher photocurrent and faster response rate..$^{9,28}$

\section{Conclusions}

In summary, a simple solvothermal procedure is demonstrated to prepare $\mathrm{SnS} / \mathrm{RGO}$ hybrid nanosheets. The large-size photodetectors based on the nanocomposites exhibits an excellent photoresponsivity of $0.18 \mathrm{~A} \mathrm{~W}^{-1}$ under visible-light illumination with a detectivity of $4.18 \times 10^{10}$ Jones. The SnS/RGO hybrids thus show promising potential for cost effective, environmental friendly, less toxic, and naturally occurring optical, electronic, and optoelectronic devices.

\section{Conflicts of interest}

There are no conflicts to declare. 


\section{Acknowledgements}

This work was supported by the Natural Science Foundation for Young Scientists of Gansu Province, China (Grant No. 1506RJYA220), the National Natural Science Foundation of China (Grant No. 51335010), the Fundamental Research Funds for the Central University (lzujbky-2017-175 and lzujbky-2016116), the Lanzhou Chengguan District Scientific Reaserch Foundation (cgkj2015-5-1).

\section{Notes and references}

1 A. Ferrari, F. Bonaccorso, V. Fal'ko, K. Novoselov, S. Roche, et al., Nanoscale, 2015, 7, 4598-4810.

2 H. Chen, H. Liu, Z. Zhang, K. Hu and X. Fang, Adv. Mater., 2016, 28, 403-433.

3 K. Hu, H. Chen, M. Jiang, F. Teng, L. Zheng and X. Fang, Adv. Funct. Mater., 2016, 26, 6641-6648.

4 X. Fang, L. Hu, K. Huo, B. Gao, L. Zhao, M. Liao, P. K. Chu, Y. Bando and D. Golberg, Adv. Funct. Mater., 2011, 21, 39073915.

5 J. Chao, S. Xing, Z. Liu, X. Zhang, Y. Zhao, L. Zhao and Q. Fan, Mater. Res. Bull., 2018, 98, 194-199.

6 Z. Sun, Z. Liu, J. Li, G. Tai and S. Lau, Adv. Mater., 2012, 24, 5878-5883.

7 D. Son, B. Kwon, D. Park, W. Seo, Y. Yi, B. Angadi, C. Lee and W. Choi, Nat. Nanotechnol., 2012, 7, 465-471.

8 D. Kufer, I. Nikitskiy, T. Lasanta, G. Navickaite and F. Koppens, Adv. Mater., 2015, 27, 176-180.

9 L. Gao, C. Chen, K. Zeng, C. Ge, D. Yang, H. Song and J. Tang, Light: Sci. Appl., 2016, 5, e16126.

10 S. Li, J. Zheng, S. Zuo, Z. Wu, P. Yan and F. Pan, RSC Adv., 2015, 5, 46941-46946.

11 Z. Deng, D. Cao, J. He, S. Lin, S. M. Lindsay and Y. Liu, ACS Nano, 2012, 6, 6197.

12 J. Chao, Z. Xie, X. B. Duan, Y. Dong, Z. R. Wang, J. Xu, B. Liang, B. Shan, J. H. Ye, Di Chen and G. Z. Shen, CrystEngComm, 2012, 14, 3163.
13 J. Chao, Z. Wang, X. Xu, Q. Xiang, W. Song, G. Chen, J. Hu and D. Chen, RSC Adv., 2013, 3, 2746-2753.

14 T. S. Reddy and M. C. S. Kumar, $R S C A d v ., 2016$, 6, 9568095692.

15 M. S. Mahdi, K. Ibrahim, A. Hmood, N. M. Ahmed, S. A. Azzez and F. I. Mustafa, RSC Adv., 2016, 6, 114980114988.

16 F. Lu, J. Yang, R. Li, N. Huo, Y. Li, Z. Wei and J. Li, J. Mater. Chem. C, 2015, 3, 1397-1402.

17 M. S. Mahdi, K. Ibrahim, A. Hmood, N. M. Ahmed, F. I. Mustafa and S. A. Azzez, Mater. Lett., 2017, 200, 10-13.

18 J. Wang, G. Lian, Z. Xu, C. Fu, Z. Lin, L. Li, Q. Wang, D. Cui and C. Wong, ACS Appl. Mater. Interfaces, 2016, 8, 9545-9551.

19 X. Zhou, Q. Zhang, L. Gan, H. Li, J. Xiong and T. Zhai, Adv. Sci., 2016, 3, 1600177.

20 X. Zhou, L. Gan, Q. Zhang, X. Xiong, H. Li, Z. Zhong, J. Han and T. Zhai, J. Mater. Chem. C, 2016, 4, 2111-2116.

21 J. Xia, D. Zhu, X. Li, L. Wang, L. Tian, J. Li, J. Wang, X. Huang and X. Meng, Adv. Funct. Mater., 2016, 26, 4673-4679.

22 Z. Jia, S. Li, J. Xiang, F. Wen, X. Bao, S. Feng, R. Yang and Z. Liu, Nanoscale, 2017, 9, 1916-1924.

23 S. S. Shinde, A. Sami, D. Kim and J. Lee, Chem. Commun., 2015, 51, 15716-15719.

24 X. An, J. C. Yu and J. Tang, J. Mater. Chem. A, 2014, 2, 10001005.

25 D. Zhang, L. Gan, Y. Cao, Q. Wang, L. Qi and X. Guo, Adv. Mater., 2012, 24, 2715-2720.

26 P. Yu, X. Yu, W. Lu, H. Lin, L. Sun, K. Du, F. Liu, W. Fu, Q. Zeng, Z. Shen, C. Jin, Q. Wang and Z. Liu, Adv. Funct. Mater., 2016, 26, 137-145.

27 Y. Fu, G. Gou, X. Wang, Y. Chen, Q. Wan, J. Sun, S. Xiao, H. Huang, J. Yang and G. Dai, Appl. Phys. A, 2017, 123, 299.

28 X. Zhou, Q. Zhang, L. Gan, H. Li and T. Zhai, Adv. Funct. Mater., 2016, 26, 4405-4413. 